\title{
The Visual Display of Temporal Information for E-Textbook: Incorporating the Mind-mapped Timeline Authoring Tool
}

\author{
HeeJeong Lee ${ }^{1 *}$ and Kok-Lim Alvin Yau ${ }^{2}$ \\ ${ }^{1}$ Pierson College, PyeongTaek University \\ Pyeongtaek-si, Gyeonggi-Do, 450-701 - Korea \\ [e-mail: hjlee@ptu.ac.kr] \\ ${ }^{2}$ School of Science and Technology, Sunway University \\ Bandar Sunway, 47500 - Malaysia \\ [e-mail: koklimy@sunway.edu.my] \\ *Corresponding author: HeeJeong Lee
}

Received December 7, 2017; revised January 29, 2018; accepted February 20, 2018;

published July 31, 2018

\begin{abstract}
With the ever-increasing queries related to temporal (or time-related) information, such as the product launching time, in search engine, most web pages will be augmented with such information in the future. Meanwhile, the gradual emergence of the use of electronic textbooks (or e-Textbooks), which enrich the traditional paper-based textbooks with multimedia contents such as interactive quizzes and multimedia-based simulations, has led us to infer that e-Textbooks will be blended with temporal information to support learning. The use of temporal information helps teachers and students to understand the level of prior knowledge required to study a topic, as well as the sequence of learning activities and related sub-topics, that best attains the educational goals. This paper presents a simple yet efficient tool called TimeMap, which is based on mind mapping, to create an e-Textbook called TimeBook that takes account of time-related curriculum and the ability of students to learn via collaboration.
\end{abstract}

Keywords: Electronic textbook, authoring tool, digital textbook, mind map, temporal information, open education resource

A preliminary version of this paper appeared in the 41st ASEE/IEEE Frontiers in Education Conference, October 12 - 15, 2011, Rapid City, USA. This version includes Wikipedia and Wikibooks containing temporal information so that users can search a Wikipedia repository and make use of a collaborative learning environment where teachers and students can work together. 


\section{Introduction}

Temporal (or time-related) information relates a state or an action with a timestamp. Temporal information has found its importance in various tasks, including information extraction, topic tracking, concept linkage, and Web search. A timeline is a linear representation of important events in the order in which they occur, and it is important to display the progression of knowledge from fundamental to advanced levels.

A textbook is a repository of educational content that contains detailed information about a subject matter. Suppose, a grade nine student wishes to learn about a topic called functions in a mathematics textbook. The student must know prior knowledge about this topic (e.g., functions at grade eight), and should be aware of existing and future knowledge about this topic (e.g., functions at the university level). Both prior and future knowledge can be obtained through the extrapolation of the timeline for the scope of a topic. Hence, the time values for the past, present and future contexts are of great interest.

Electronic textbooks (e-Textbooks) are "digitized forms of textbooks which are envisioned to replace existing paper-based textbooks" [1]. Compared to traditional textbooks, four main advantages of e-Textbooks have been reported in the literature [2][3]. Firstly, the incorporation of multimedia contents, such as video clips, animations and education-based games, has shown to capture students' interest in study. Secondly, students are no longer carrying backpacks loaded with heavy paper-based textbooks to schools. Thirdly, e-Textbooks enable easy backup and replacement because any losses can be easily replaced by new copies from e-Textbook servers. Fourthly, fast update and access to the latest content are possible. Despite the popularity of e-Textbooks in carrying out pedagogical activities, there is lack of research interest to embed temporal information into e-Textbooks, although using such information to generate the content of e-Textbooks is valuable for curriculum planning and learning.

A learning model is a structural and conceptual foundation and framework that outlines the learning activities and environments in which students learn and develop their skills, all of which are inline with educational goals and promoted by government initiative. Hence, a learning model is analogous to a blueprint of the curriculum that guides the selection and arrangement of learning experiences [4]. For example, Mathematics must be taught in a logical sequence, progressing from fundamental knowledge (e.g., integer and real number) to intermediate (e.g., summation and subtraction) to advanced (e.g., differentiation and integration) levels. A student who has missed a lesson, or failed to grasp a concept, which serves as a prerequisite to the next level of study, may become handicapped in understanding the concepts and knowledge in subsequent classes.

We have developed a timeline-based e-Textbook called TimeBook [5] that incorporates temporal information using a TimeMap authoring tool. As the knowledge that students gain evolves with time, TimeMap helps students from grade levels K1 to K12 to understand the required prerequisite knowledge they are lack of before proceeding to the next chapter or level of study, and subsequently it generates TimeBook covering the prerequisite knowledge using study materials obtained from relevant resources that may include text, videos and images. TimeMap makes it possible for students to take a preview and an overview of what they will learn, as well as what they have missed, using an e-Textbook. 
For visualization, TimeMap uses the mind mapping concept to generate an illustration for the teaching and learning framework. It allows users to create concept nodes and connect among themselves in order to organize, classify and generate a layout information [6][7][8]. While there are a number of popular mind mapping software, such as Concept Draw MINDMAP 5 Professional [9], XMIND [10], MindManager [11], and FreeMind [6], TimeMap is unique as it embeds temporal information, particularly the time frame (or sequence), into the concept nodes and their connections. For instance, in TimeMap, the grade levels are represented by time frame (or sequence). Teachers can use TimeMap to plan and develop a learning model; while students can use it to develop their customized e-Textbooks based on their individual mastery of the prerequisite knowledge. TimeMap not only demonstrates a greater use of study resources, but also indicates the important directions in developing a learning model.

Although the concept and framework of TimeMap are applicable to different applications in which temporal information is of paramount importance, we focus on the use of temporal information to generate e-Textbooks for curriculum planning. The contribution of this paper is to describe a novel and interactive authoring tool, namely TimeMap, for teaching and learning, allowing teachers and students to generate TimeBook, which is an e-Textbook augmented with temporal information. The temporal information is valuable for curriculum planning and learning, allowing users to easily contrast and observe the evolution of a topic of interest over a timeline. We highlight the evolution of time in creating e-Textbooks, which has great potential to enhance teaching and learning approaches.

The rest of this paper is organized as follows. Section 2 presents related work. Section 3 shows the incorporation of temporal information into TimeBook using TimeMap. Section 4 demonstrates temporal query processing. Section 5 presents conclusions and future work.

\section{Related Work}

In this section, we present an overview of research works on the visualization of temporal information, and the potential use of Wiki as a teamwork platform for collaborative e-Textbook development.

\subsection{Visualization of Temporal Information}

Software implementation of the visual representation of temporal information enables the relationship between time and the dynamics of events and actions to be shown. Traditionally, the space time cube approach "combines time and space in a single display", whereby the horizontal dimension represents a two-dimensional space and the vertical dimension represents time, giving a geographical expression of time [12][13]. One of the earliest usage of the space time cube approach was to develop a socio-economic web model by Hägerstand in 1970s [14]. We review a range of space time cube techniques, namely time cutting, time coloring, and time labeling.

Time cutting extracts and displays a time slice, which is a snapshot of a two-dimensional space from a cube at a particular time instance [14]. In general, a video can be represented using a spatio-temporal cube whereby a series of time slices (or pictures) at different time instances are piled to form a cube. Hence, time cutting captures, extracts and displays a particular time slice in a video. Nevertheless, time cutting can capture, extract and display a single time slice at a particular time instance only; while in e-Textbooks, a single time slice 
should be viewed multiple times. For instance, a single topic may be taught at different grade levels using different content resources.

Time coloring changes the color of each time slice over time [14]. The color of each time slice can be specified according to a linear color scale. The temporal information can be displayed by changing the hue of each time slice or by applying a discontinuous color based on the ages of the events. Due to the large number of possible overlapping colors, using colors as representation can be scalable for a large data set with a large variety of information; however, the temporal information must be readable regardless of the screen size of a device (i.e., a smartphone).

Time labeling adds a time label, which contains the starting time or the time period of an event (e.g., a grade level) [15], to each time slice, or to an object within a spatio-temporal volume. Similarly, in this work, TimeMap uses time keys with different colors (see Section 3.1) and numbers (see Fig. 5) to represent the time period of an activity.

Nevertheless, the space time cube approach can become illegible as it can be visually cluttered with information [16], which can reduce the number of paths (or progressions) that can be effectively explored, the length of the time period, and the capacity to represent various aspects of dynamics. As TimeMap associates a time label with each activity or topic in a space-time volume for temporal data visualization, a time axis is not necessary, which helps to minimize visual cluster in TimeMap.

Next, Cousins and Kahn have presented a formal system for representing time-ordered patient data using timeline [17]. The system provides an interactive environment that helps users to visualize temporal data. However, temporal uncertainty has not been incorporated due to the imprecision of temporal measurements and temporal knowledge. For example, two events may occur at the same time (i.e., on the same day); however, the two events would have occurred at different times if a finer temporal granularity (e.g., at the minute or hour level) is adopted. As events are often imprecisely dated in practice [18], using a fine temporal granularity may not provide accurate temporal information. To overcome this, TimeMap provides the flexibility to adjust temporal granularity, as well as the capacity of various resources.

Lastly, Stojanovic et al. have visualized temporal information for different events using computer oriented techniques [15]. In particular, "the system can show the time that an event commences or the period of its existence through labels". The ages of the events are represented by different colors. Similarly, TimeMap uses time keys, which have different colors, to represent the age of an activity (see Section 3.1), as well as numbers (see Fig. 5), for better representation.

\subsection{Transforming Wiki Sources to Structured Extensible Markup Language (XML)}

A Wiki system, such as Wikipedia, is an important online collaboration platform that enables web-based knowledge sharing and provides content repositories [19]. It is equipped with a real time editor supported by Wiki markup (WikiML), which is a language similar to but less expressive (see Table 1) than HTML [20]. As "Wiki can be easily created using popular software, such as wikispaces.com and MediaWiki” [21], it can serve as a platform for collaborative learning. MediaWiki has been adopted by TimeMap to provide a collaborative platform that serves as a content repository for content providers, developers and end users.

Each Wiki article comprises of a title, content, and links [22]. To use the content of a Wiki directly while authoring content for e-Textbooks, transformation from WikiML into XML is 
needed because TimeMap and TimeBook are expressed in XML. An example of a transformation from WikiML to XML is shown in Table 1, and it shows a simple Wiki article containing three different levels. In this example, an equal sign (“=”) represents a header for a different level.

Table 1. Transforming mediaWiki markup to xml format

\begin{tabular}{|c|c|}
\hline WikiML & XML \\
\hline $\begin{array}{c}==\text { Level } 2 \text { == } \\
===\text { Level } 3 \text { === } \\
====\text { Leverl } 4====\end{array}$ & $\begin{array}{l}\text { section }> \\
\quad<\text { title }>\text { Level } 2<\text { /title }> \\
<\text { section }> \\
\quad<\text { title }>\text { Level } 3<\text { /title }> \\
\quad<\text { section }> \\
\quad<\text { title }>\text { Level } 4<\text { /title }> \\
\quad<\text { /section }> \\
\quad<\text { /section }> \\
\text { </section }>\end{array}$ \\
\hline
\end{tabular}

\section{Implementation of Incorporating Temporal Expressions in e-Textbooks}

This section presents the implementation of incorporating temporal expressions in e-Textbooks. There are four main steps: 1 ) creating a linear timeline, 2) converting timelines to a mind map, 3) converting a mind map to XML, and 4) converting XML to an e-Textbook. Finally, a temporal query processing is illustrated. For clarity, the four main steps are presented along with an example for the functions topic in the Mathematics subject.

\subsection{Representation of a TimeBook}

TimeBook [5] has four-tuple information ( $T, L, A, R)$ :

- Topic $(T)$ is the knowledge taught based on the syllabus. An e-Textbook may contain a number of topics. Each topic may contain a collection of timelines representing the actual timestamp of the occurrence (e.g., grade level) of a task, a scope, and a sequence of instructions, and must contain at least a single timeline (e.g., a series of grade levels). A topic taught in several grade levels must appear in several timelines.

- Timeline $(L)$ is a graphical or textual representation of a period of time, and it allows events to be marked in a chronological order [23]. It enables readers to understand the relationship of events with respect to time. As an example, for a topic called functions, input/ output tables and function tables are covered in grade four, linear functions are covered in grade five, as well as linear and nonlinear functions are covered in grade six. While the same topic is taught in several grade levels, the learning outcomes for each grade level are different. Since a topic may consist of a number of timelines, the time keys (i.e., the grade levels) are kept within a topic (see Fig. 1).

- Activity $(A)$ includes the general and specific learning plans or achievement indicators. Each topic may consist of a set of activities, and so they are represented in different timelines. As an example of the topic called functions, input/output tables with addition, subtraction, multiplication, and division activities are covered in grade four. Each activity is represented by a unique name and a time key (e.g., K4, K5, or K12). 
- Resource $(R)$ includes texts, images, videos or quizzes that teachers use to enhance and support the effectiveness of the learning process to meet the expectations of learning as defined by syllabus. Each activity is allocated with resources.

Fig. 1 shows an example of a graph that consists of a topic (i.e., functions) and a timeline. The functions topic has different activities, including function table, linear functions, as well as linear and nonlinear functions. These activities have different time keys (i.e., K4, K5 and K6). The topic and timeline help to organize the activities of a topic and facilitate meaningful learning of a subject matter.

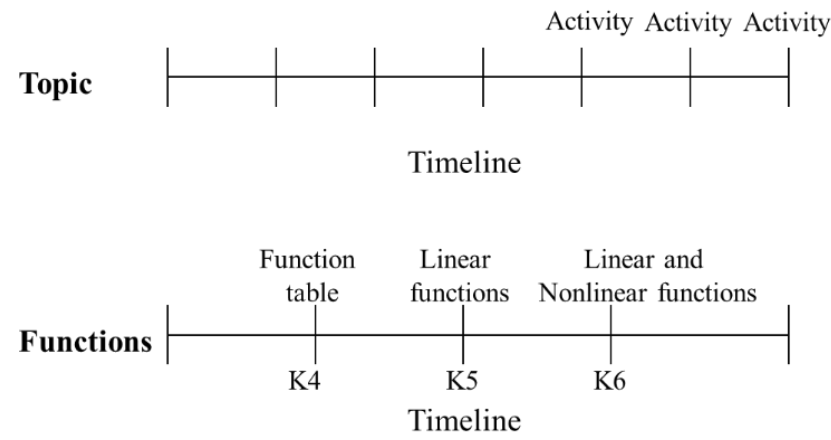

Fig. 1. Topic and timeline for a generic topic and a topic called functions, respectively.

\subsection{Conversion from a Timeline to a Mind Map}

A linear timeline (see Fig. 1) illustrates the activities of a topic taught in a certain grade level. Using a linear timeline has some limits. In linear arrangement, activities are "placed successively on higher level on the screen and they are placed further right in accordance with its beginning and ending time" [23]. So, if an activity has been forgotten to be listed, it is difficult to include it later as the sequence of the activities is linear in nature (e.g., K6 activities happen after K4 activities). Converting a timeline to a mind map provides users with the capability to handle a complex structure containing a large amount of temporal information.

We show how to add a topic, followed by activities, resources, and finally a timeline to a TimeMap. Teachers and students can add any number of branches and sub-branches to a mind map to represent different topics and activities. Fig. 2 shows an example of a mind map for the Mathematics subject. In Fig. 2, the main branches (e.g., polynomials, rational functions) are the topics, and the sub-branches (e.g., linear, quadratic or cubic) are the subtopics or activities.

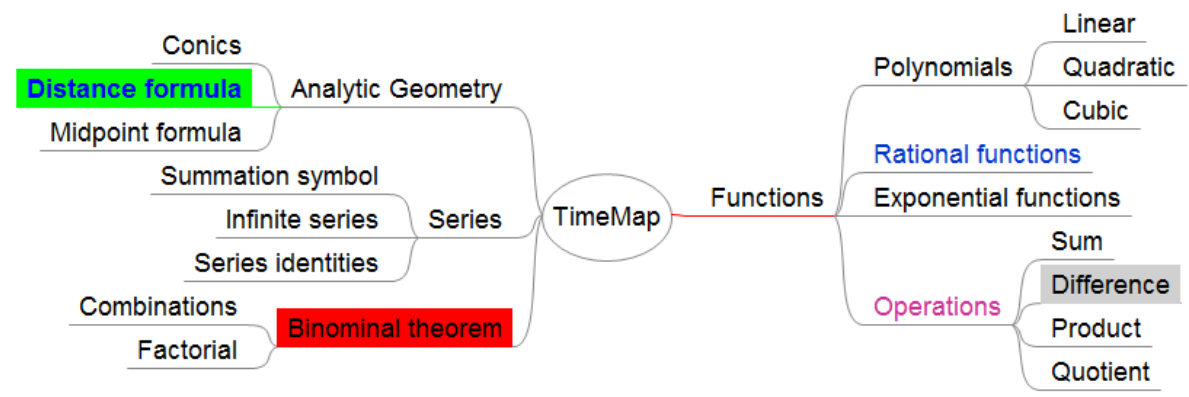

Fig. 2. An example of a mind map for the Mathematics subjects. 
Users can decide the information that a timeline shows, such as topics, activities related to a topic, the time period of a timeline, and resources related to a topic. Users can add main branches and sub-branches by selecting New Child Node as shown in Fig. 3. When constructing topics, one proceeds from general topics to specific ones, from the abstract to the concrete [24]. Different fonts and colors can be used.

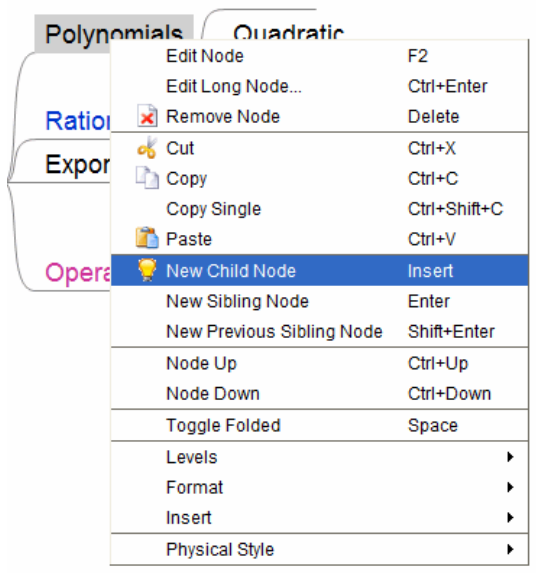

Fig. 3. Adding a subtopic by adding a new child node called polynomial for a main topic called functions.

Fig. 4 shows an example of adding teaching and learning resources, in particular the external resources such as images and Wikipages, to the concept nodes. In this way, each activity may have links to resources, which appear as icons (e.g., pencil, movie clip, and arrow) in TimeMap. Interested users can access the resources by clicking these icons (see Fig. 6).

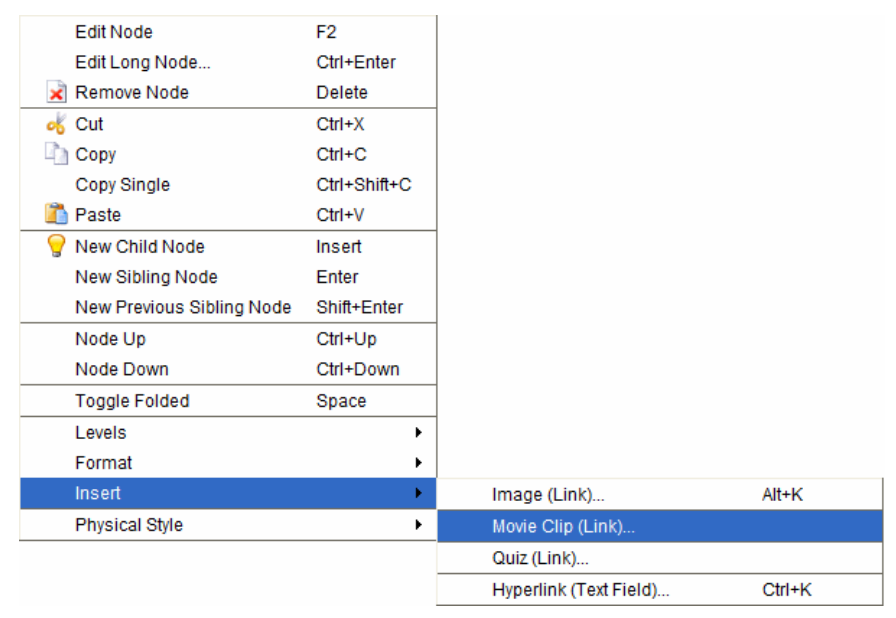

Fig. 4. Adding teaching and learning resources.

Fig. 5 shows an example of adding grade levels using time key icons. By this way, a node has a timeline. The number shown in an icon indicates a time instance (e.g., semester, grade, and year), allowing a timeline to be divided into grade levels. A higher value indicates a higher level (i.e., the level of knowledge), and a lower value indicates a lower level. Additionally, the unit of the timeline can be flexible. Hence, a grade level can be adjusted to reflect an annual or a semester basis. With smaller units, the timeline becomes more precise. 


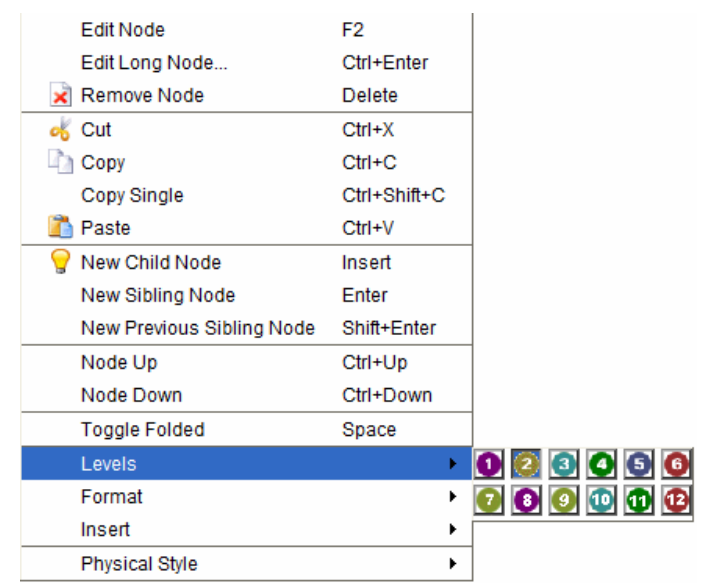

Fig. 5. Adding time key icons. A number represents a time key, which is a grade level in this case; for instance, the number 1 represents K1, 2 represents K2, and so on.

Fig. 6 illustrates the resulting TimeMap consisting time key icons and resources for a Mathematics subject. The relationship among the topics in Mathematics can be visualized using TimeMap "in a structured way that corresponds to the structure in mathematics" [24].

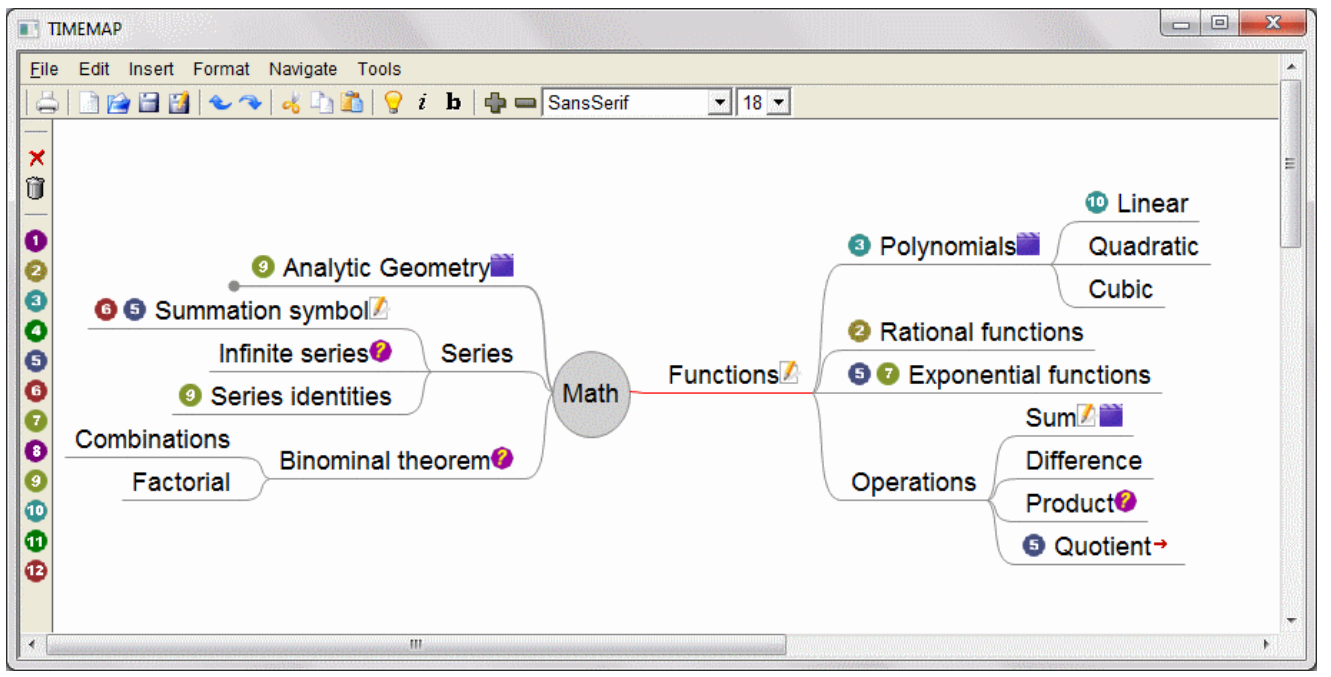

Fig. 6. The resulting TimeMap for the Mathematics subject. Icons (i.e., pencils) represent links to resources and numbers represent time keys.

\subsection{Conversion from a Mind Map to XML}

TimeMap uses a graphical representation for topics or learning activities. The output file from TimeMap is an XML file that follows "a classical graph data structure, where each element is represented by a graph node, which is characterized by sub-elements such as text, font, icon, diagram position, among others” [25]. Using XML file allows easy transition to other mind mapping software such as FreeMind. Data generated in the XML file can also be exported to an e-Textbook XML document or an external XML database. 


\subsection{Conversion from XML to an e-Textbook}

TimeMap parses resources and information embedded in an XML file to create an interactive e-Textbook called TimeBook as shown in Fig. 7. In Fig. 7, the navigation buttons allow a user to turn to the next page or previous page, or type in a page number so that the book can flip to the page indicated. Generally speaking, an e-Textbook reads an XML file, and subsequently loads and displays the images, texts and movie clips.

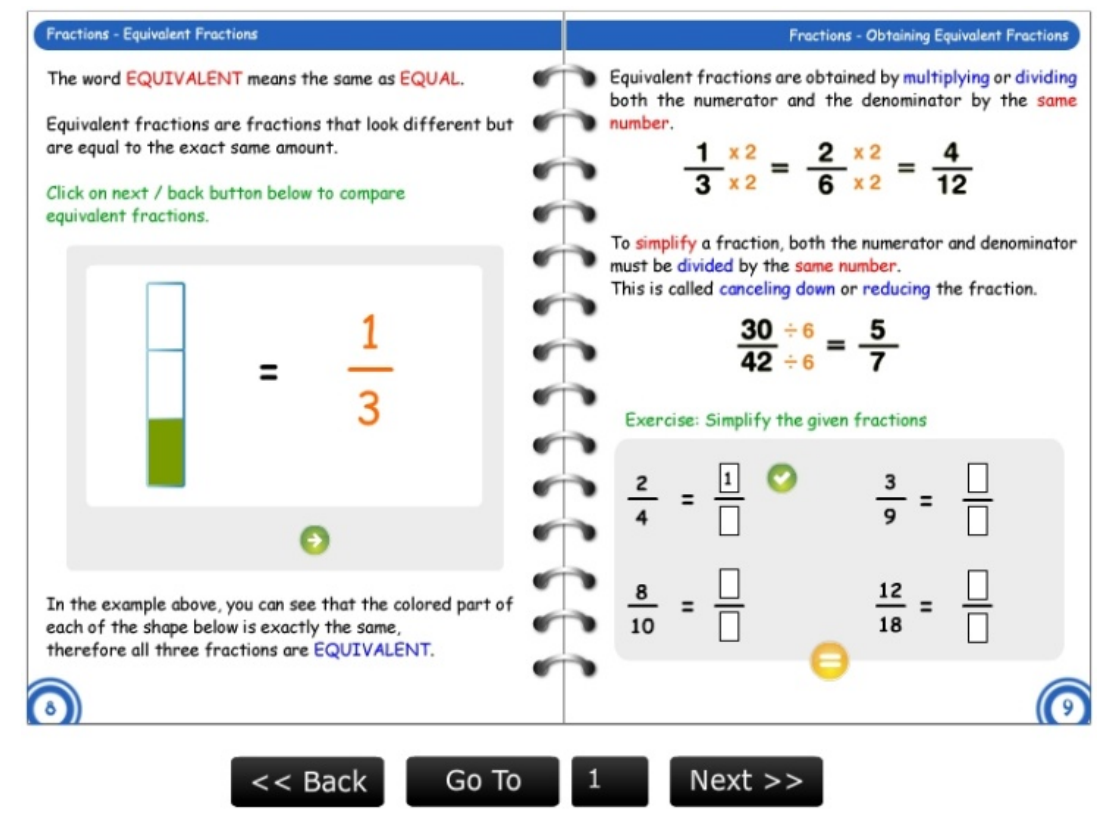

Fig. 7. An example of a TimeBook for the functions topic.

\subsection{Implementation}

As mind map requires visual cues for its implementation, FreeMind is used as part of our mind map design. FreeMind is a free mind mapping software written in Java [26], although it can be modified using JavaScript, HTML and AJAX. Apart from general mind map features, our TimeMap has the following features:

- HTML links, such as www links or links to local files or resources (e.g., text, video, image, quiz, and flash game), are stored in the nodes.

- Mind maps can be exported to e-Textbook in XML format.

- Nodes can be embedded with built-in grade level icons (e.g., K1-K12), colors and different fonts.

Users can use TimeMap for the following purposes:

- Keeping track of learning models, which can be very dynamic, while recording subtopics at different grade levels.

- Keeping a collection of resources with links in order to expand the content of the topics presented in an e-Textbook. 


\section{Temporal Query Processing}

\subsection{Open Educational Resources}

Suppose, each author has to develop individual curriculum-related educational resource to create e-textbooks, this would require a large amount of effort which can be a great burden. A collection of learning resources held at a central location can help all participants to share resources among themselves [27]. Open educational resources (OER) allows "digitized materials offered freely and openly for educators, students, and self-learners to use and reuse for teaching, learning, and research. OER includes learning content, software tools to develop, use, and distribute content, and implementation resources such as open licenses”. As OER is freely available to be used, mixed, updated, and redistributed, it is supported by contributions from government agencies, scholars and educationists around the world. There is an open-source textbook project at Connexions [28], which works under the "Creative Commons" attribution license. It provides search for users by grade, subject area, and content. Teachers can select modules to be included in their course, and the content is freely available. Flexbook [29] and ReadWriteThink [30] are other examples of OER sites [27].

\subsection{An Example of Temporal Query Processing}

In this section, for clarity, we show an example of how OER content can be edited to insert time keys. MediaWiki is used to serve as a content repository as it allows Wikipedia raw data, which is an online encyclopedia, to be exported in XML via its API. Subsequently, TimeMap can be used to retrieve the data, and transform the data to produce an . $\mathrm{mm}$ file, which can be subsequently edited using a mind map tool. Users can use a search form to search for a keyword in a Wikipedia repository, and TimeMap can be used to show a list of articles containing the keyword. Such search tool, which is written in JavaScript using Ajax technologies, shows a list of search keywords while a keyword is being keyed in, and it is integrated into TimeMap user interface (see Fig. 8). Consider keying in a machine keyword in the search box, a list of search keywords, including machine learning, is shown. Suppose, this keyword is selected, the search outcome is shown in Fig. 9 in the form of a mind map.

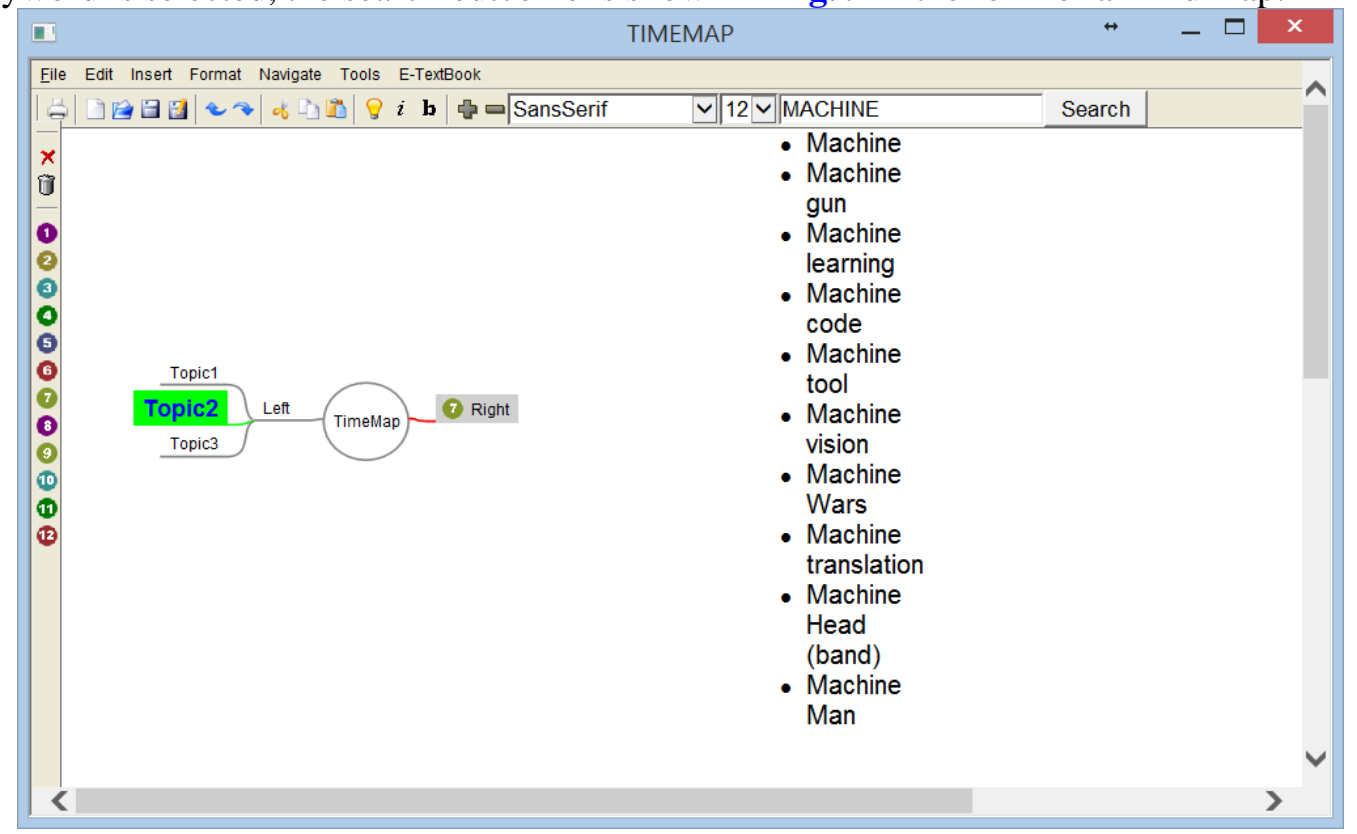

Fig. 8. Searching teaching and learning resources. For a machine learning topic, a machine keyword in the search box produces a list of search keywords, including machine, machine gun, and so on. 
TimeMap converts raw data into XML for display in TimeMap in the form of a mind map (see Fig. 9). In the raw data, headers are identified by a double "=" signs at the start and the end of each header title (see Table 1). Using the extracted raw data, TimeMap displays subtopics and URLs to update its contents rapidly using the AJAX technology. Unlike TimeMap (see Fig. 6), in Fig. 9, hyperlinks to the content are provided without any notion of time. Nevertheless, using mind map, subtopics are displayed visually.

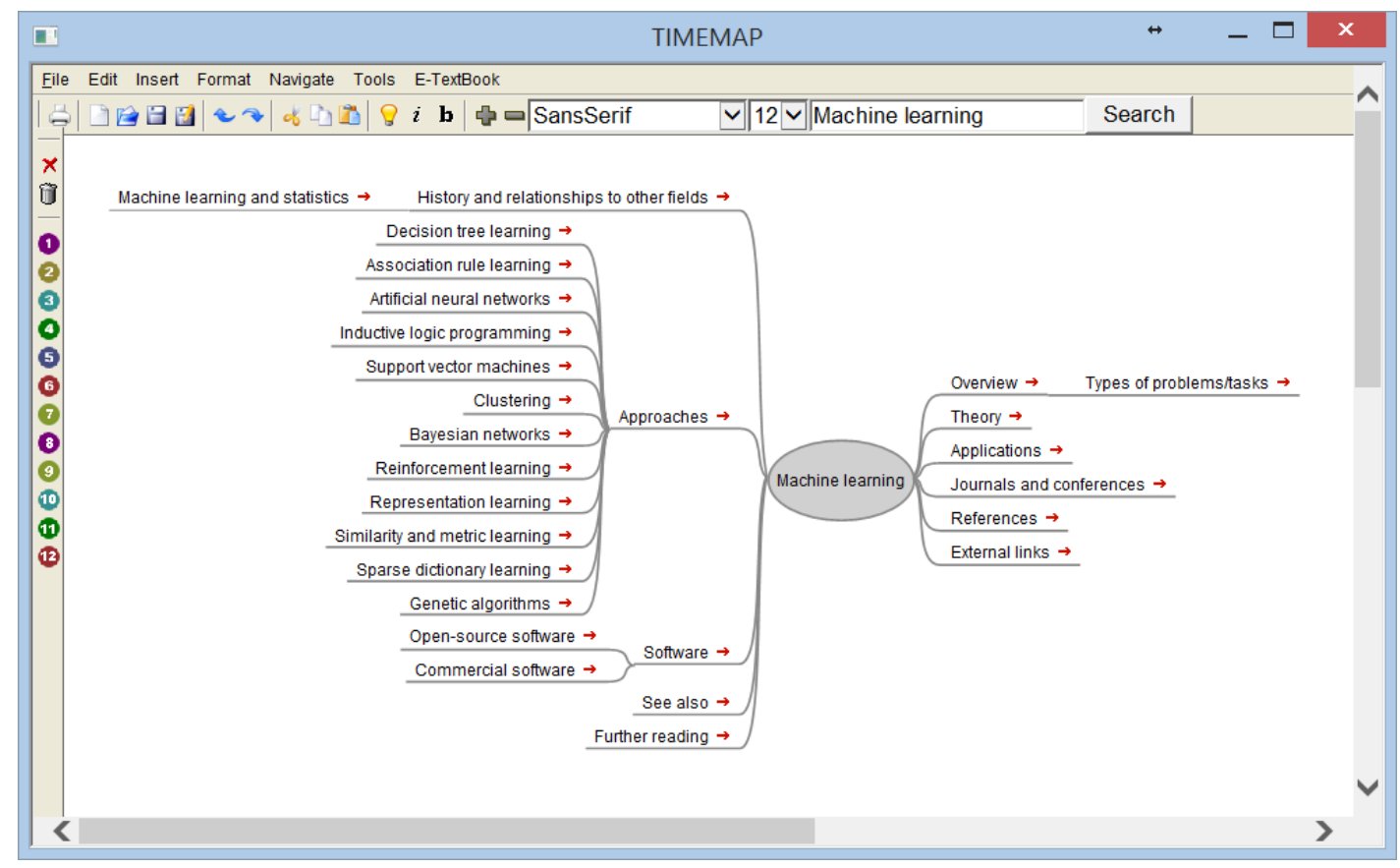

Fig. 9. Displaying topics with links. Icons (i.e., arrows) shown beside the subtopics and activities, represent links to resources.

Once a mind map has been created, time-dependent information is added to the topics and subtopics. Fig. 10 illustrates the inclusion of time keys using icons. In this way, the nodes have temporal information. 


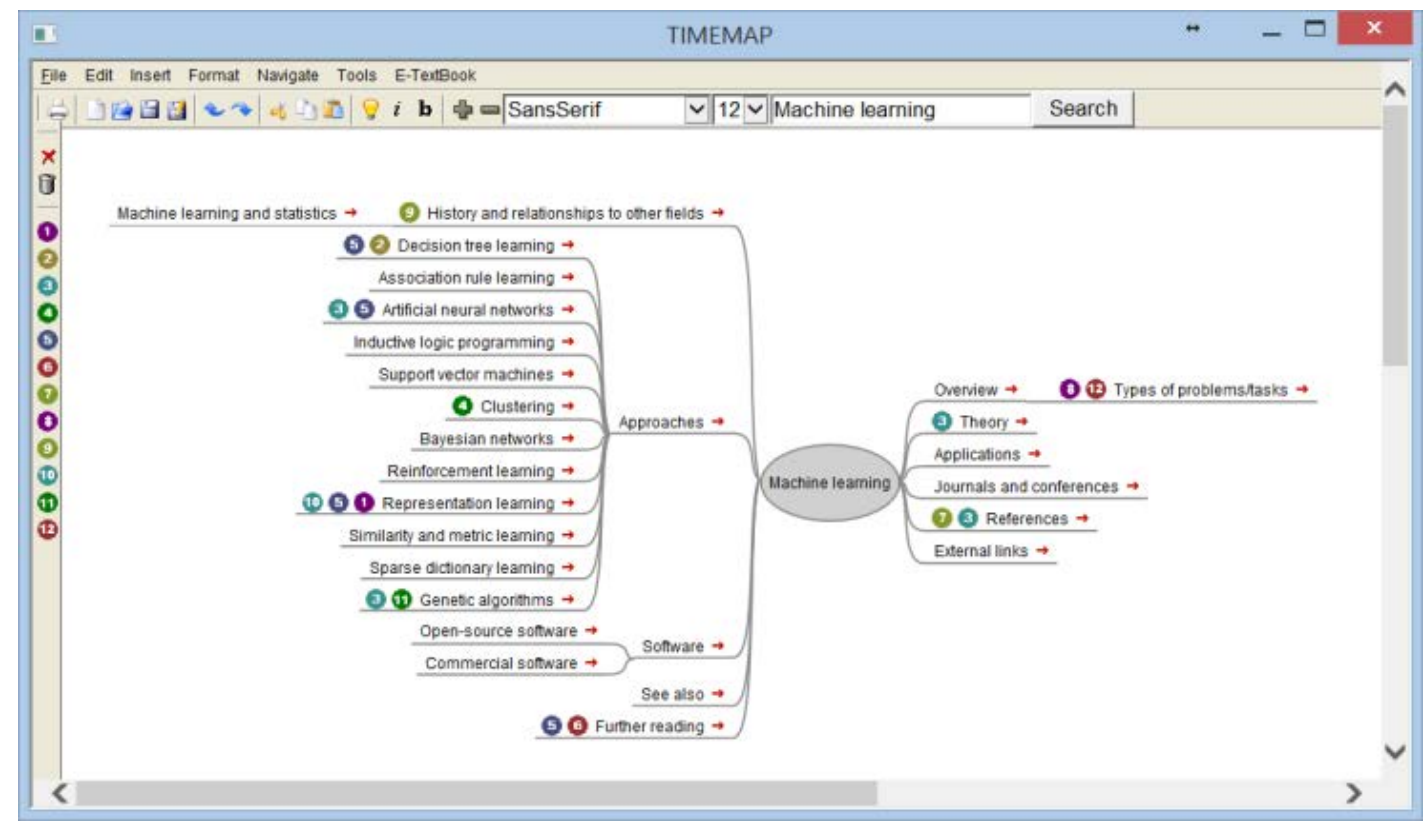

Fig. 10. Adding time key icons to provide grade levels for subtopics.

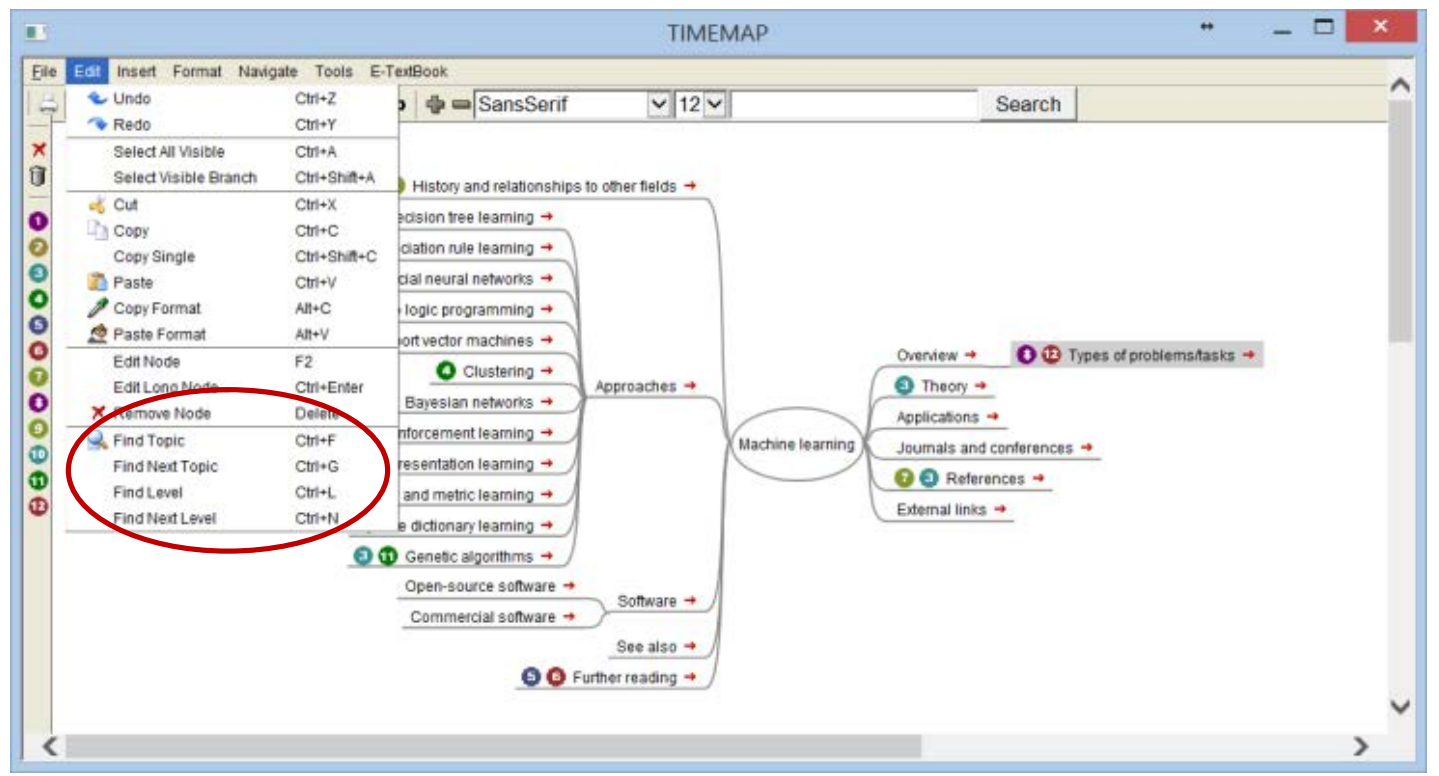

Fig. 11. Querying a time key. Red circle shows various kinds of searches that allow users to search with respect to topics and time keys.

Querying software program is "capable of answering users' questions concerning data under analysis" [31]. When users search for topics, they can see the timeline from the root node to the leaf node. Information can be searched based on the topic and the level of constraints (see Fig. 11).

The TimeMap can be saved as an XML file and reopened to present a mind map with time keys. MediaWiki platform is used to keep contents and resources for e-Textbooks via services that enhance contents with temporal metadata. The use of MediaWiki platform for storage, retrieval and reuse of teaching and learning resources has the following advantages: 
- Contents can be created and shared with other users.

- Teaching and learning contents can be embedded with online resources, such as integrating OER into e-Textbooks.

- A collaborative and familiar learning environment, in which teachers and students can work together, can be established.

In short, the successful use of TimeBook depends on the collaborative aspects, the simplicity in creating and interlinking the contents, documents and time key icons, as well as the openness of reading and editing.

\section{Conclusions and Future Work}

In this paper, we have attempted to use temporal information, which is valuable for curriculum planning and learning, for curriculum generation in e-Textbooks. We present our prototype, called TimeMap and TimeBook, to visualize the temporal information in e-Textbooks, and suggest collaborative learning via Wiki-based e-Textbooks. To show the effectiveness of our approach, Wikipages are used to display time information using mind map. By adding temporal information into e-Textbooks, a clearer picture of the learning activities can be provided. The use of MediaWiki to facilitate the sharing of learning resources is also presented.

In our future work, we aim to improve TimeMap with three main enhancements. Firstly, TimeMap can provide query results with multiple time keys or intervals (e.g., K5 and K6, as well as K5 - K7). Secondly, TimeMap filters out the contents that do not satisfy the query constraints so that such contents are not displayed. Thirdly, the visual display of spatio-temporal curriculum planning and learning can be enhanced to process not only temporal information, but also dynamic spatial data.

\section{References}

[1] H. J. Lee, C. Messom, and A. Y. Kok-Lim, "Can an electronic textbooks be part of K-12 education?: Challenges, technological solutions and open issues," TO-JET: The Turkish Online Journal of Educational Technology, vol. 12, pp.32-44, Jan. 2013. Article (CrossRef Link)

[2] G. Ozsoyoglu, N. H. Balkir, Z. M. Ozsoyoglu, and G. Cormode, "On Automated Lesson Construction from Electronic Textbooks," IEEE Transactions on Knowledge and Data Engineering, vol. 16, pp. 317-331, Mar 2004. Article (CrossRef Link)

[3] H. J. Lee and K.-L. A. Yau, "Addressing the major information technology challenges of electronic textbooks," Journal of Computer Information Systems, vol. 55, pp. 40-47, Dec. 2015. Article (CrossRef Link)

[4] M. G. E. Svensson, "System Dynamics of Learning Processes: Comparing Apples with Pears," in Proc. of Proceedings of the system dynamics conference of the system dynamics society, Palerno, Italy, May. 2002. Article (CrossRef Link)

[5] H. J. Lee, T. Ong, and C. H. Messom, "Timeline-based authoring tool for e-Textbook: Bringing the "mind-mapped learning model," in Proc. of Proceedings of the Frontiers in Education Conference (FIE), Rapid City, SD, USA, Oct. 2011, pp. S1E-1-S1E-5. Article (CrossRef Link)

[6] Calvo, U. Rueda, J. A. Elorriaga, A. Arruarte, and M. Larranaga, "Idea Map Editor: a tool for promoting reflection in the act of learning," in Proc. of Proceedings of the Ninth IEEE International Conference of the Advanced Learning Technologies, Riga, Latvia, pp. 361-363, Jul. 2009. Article (CrossRef Link)

[7] H. Dagez and K. Hashim, "Mind map learning approach in e-learning environment using SCORM standards," Digital Learning, Feb. 2007. Article (CrossRef Link)

[8] C. L. Willis and S. L. Miertschin, "Mind Tools for Enhancing Thinking and Learning Skills," in Proc. of Proceedings of the Information Technology Education, Newark, NJ, USA, pp. 249-254, Oct. 2005. Article (CrossRef Link) 
[9] ConceptDraw, Article (CrossRef Link)

[10] XMind, Article (CrossRef Link)

[11] MindManager, Article (CrossRef Link)

[12] T. Hägerstraand, "What about people in regional science?" Regional science, vol. 24, pp. 7-24, Jan. 1970. Article (CrossRef Link)

[13] C. Tominski, H. Schumann, G. Andrienko, and N. Andrienko, "Stacking-based visualization of trajectory attribute data," IEEE Transactions on visualization and Computer Graphics, vol. 18, pp. 2565-2574, Oct. 2012. Article (CrossRef Link)

[14] B. Bach, P. Dragicevic, D. Archambault, C. Hurter and S. Carpendale, "A Review of Temporal Data Visualizations Based on Space-Time Cube Operations," in Proc. of Proceedings of the Eurographics Conference on Visualization (EuroVis), Swansea, Wales, United Kingdom, pp. 1-19, Jun. 2014. Article (CrossRef Link)

[15] D. Stojanovic, S. Djordjevic-Kajan, A. Mitrovic, and Z. Stojanovic, "Cartographic visualization and animation of the dynamic geographic processes and phenomena," in Proc. of Proceedings of the 19th International Cartographic Conference, Ottawa, Canada, pp. 739-746, Aug. 1999, Article (CrossRef Link)

[16] G. Andrienko, N. Andrienko, and M. Heurich, "An event-based conceptual model for context-aware movement analysis," International Journal of Geographical Information Science, vol. 25, pp. 1347-1370, Jul. 2011. Article (CrossRef Link)

[17] S. B. Cousins and M. G. Kahn, "The visual display of temporal information," Artificial intelligence in medicine, vol. 3, pp. 341-357, Dec. 1991. Article (CrossRef Link)

[18] Z. Chen and F. Terrier, "About temporal uncertainty," in Proc. of Proceedings of the IEEE/ACM International Conference of the Developing and Man-aging Expert System Programs, Washington, DC, USA, Sep. 1991, pp. 223-230. Article (CrossRef Link)

[19]T. Kisner and J. Rambow, "The openFuXML Wiki Engine: Transforming Wiki Sources to Structured XML Content Objects," in Proc. of Proceedings of the Second International Conference of the Mobile, Hybrid, and On-Line Learning, Saint Maarten, Netherlands Antilles, pp. 93-98, Feb. 2010, Article (CrossRef Link)

[20] P. Kamthan, "Towards Understanding the Use of Wiki in Agile Software Development," in Proc. of Proceedings of the 28th International Conference of the Advanced Information Networking and Applications Work-shops (WAINA), Victoria, BC, Canada, pp. 566-571, May. 2014, Article (CrossRef Link)

[21] W. He and L. Xu, "Integrating both Wikis and XML with case bases to facilitate case base development and maintenance," Expert Systems with Applications, vol. 38, pp. 8632-8638, Jul. 2011. Article (CrossRef Link)

[22] I.-C. Wu, C.-H. Tsai, and Y.-H. Lin, "Clustering and summarization topics of subject knowledge through analyzing internal links of Wikipedia," in Proc. of Proceedings of the IEEE 14th International Conference of the Information Reuse and Integration (IRI), San Francisco, CA, USA, Aug. 2013, pp. 90-96, Article (CrossRef Link)

[23] E. Kursun and K. Cagiltay, "An examination of two types of timeline, linear vs. staggered: a mixed method approach," Young researchers furthering development of TEL research in Central and Eastern Europe, Nov. 2007. Article (CrossRef Link)

[24] Brinkmann, "Graphical knowledge display-mind mapping and concept mapping as efficient tools in mathematics education," Mathematics Education Review, vol. 16, pp. 35-48, May. 2003. Article (CrossRef Link)

[25]D. C. Soares, F. M. Santoro, and F. A. Baião, "eMail Mining: Knowledge intensive process discovery through e-mails," in Proc. of Proceedings of the IEEE 16th International Conference of the Computer Supported Cooperative Work in Design (CSCWD), Wuhan, China, pp. 228-235, May. 2012, Article (CrossRef Link)

[26] J. Muller, P. Novak, D. Polivaev, and C. Foltin. FreeMind -Official Homepage \& Wiki Online, Article (CrossRef Link)

[27] H. Lee, "Educational policy and planning for Future Education Development of e-Textbooks," School of Information Technology Monash University, 2012. 
[28] OpenStax CNX, Article (CrossRef Link)

[29] cK-12, Article (CrossRef Link)

[30] ReadWriteThink, Article (CrossRef Link)

[31]N. Andrienko, G. Andrienko, and P. Gatalsky, "Exploratory spatio-temporal visualization: an analytical review," Journal of Visual Languages \& Computing, vol. 14, pp. 503-541, Dec. 2003. Article (CrossRef Link)
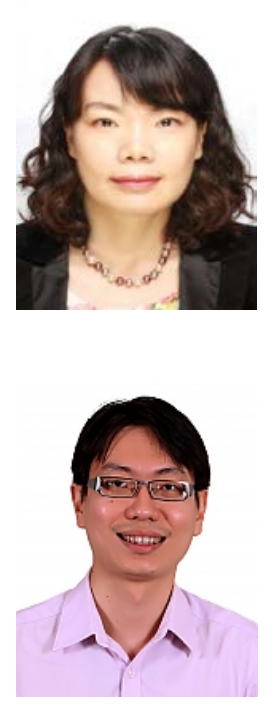

HeeJeong Lee has a BSc. (Computer Science) from Pohang University of Science and Technology (POSTECH), Korea, a MSc. (Computer Science) from the University of Edinburgh, UK, a MPhil. (Technology Policy) from Cambridge University, UK and a PhD. (Information Technology) from Monash University, Australia. She won a merit prize at a national-level competition of Asia Pacific ICT Award (APICTA'11), and a gold award at the 23rd International Invention, Innovation and Technology Exhibition (ITEX’12). Her research interests are MOOC service development, social network analysis and ICT in education. She was with Korea Telecom R\&D for 10 years; and has filed many patents on telecommunications applications. Currently, she is an assistant professor at Pierson college, PyeongTaek University.

Kok-Lim Alvin Yau has a B.Eng. degree in Electrical and Electronics (First Class Honors) from the Universiti Teknologi Petronas, Malaysia, a M.Sc. (Electrical Engineering) from the National University of Singapore, and a Ph.D. (Network Engineering) from Victoria University of Wellington, New Zealand. He was awarded the 2007 Professional Engineer Board of Singapore Gold Medal for being the best graduate of the M.Sc. degree in 2006/07. He researches, lectures and consults in ICT in education, applied artificial intelligence, and networking. Currently he is an associate professor at the Department of Computing and Information Systems, School of Science and Technology, Sunway University. 